\title{
VISIR upgrade overview: all's well that ends well
}

Florian Kerber, Hans Ulrich Käufl, Konrad Tristram, Daniel Asmus, Pedro Baksai, et al.

Florian Kerber, Hans Ulrich Käufl, Konrad Tristram, Daniel Asmus, Pedro Baksai, Nicola Di Lieto, Danuta Dobrzycka, Philippe Duhoux, Gert Finger, Christian Hummel, Derek Ives, Gerd Jakob, Lars Lundin, Dimitri Mawet, Leander Mehrgan, Eric Pantin, Miguel Riquelme, Joel Sanchez, Stefan Sandrock, Ralf Siebenmorgen, Jörg Stegmeier, Alain Smette, Julian Taylor, Mario van den Ancker, Guillermo Valdes, Lars Venema, "VISIR upgrade overview: all's well that ends well," Proc. SPIE 9908, Ground-based and Airborne Instrumentation for Astronomy VI, 99080D (3 August 2016); doi: 10.1117/12.2232441

Event: SPIE Astronomical Telescopes + Instrumentation, 2016, Edinburgh, United Kingdom 


\title{
VISIR Upgrade: all's well that ends well
}

\author{
Florian Kerber*a ${ }^{\text {a }}$, Hans Ulrich Käufl ${ }^{\mathrm{a}}$, Konrad Tristram ${ }^{\mathrm{b}}$, Daniel Asmus ${ }^{\mathrm{b}}$, Pedro Baksai ${ }^{\mathrm{b}}$, Nicola Di \\ Lieto $^{\mathrm{a}}$, Danuta Dobrzycka ${ }^{\mathrm{a}}$, Philippe Duhoux ${ }^{\mathrm{a}}$, Gert Finger ${ }^{\mathrm{a}}$, Christian Hummel ${ }^{\mathrm{a}}$, Derek Ives ${ }^{\mathrm{a}}$, Gerd \\ Jakob $^{\mathrm{a}}$, Lars Lundin ${ }^{\mathrm{a}}$, Dimitri Mawet ${ }^{\mathrm{c}}$, Leander Mehrgan ${ }^{\mathrm{a}}$, Vincent Moreau ${ }^{\mathrm{d}}$, Eric Pantin ${ }^{\mathrm{d}}$, Miguel \\ Riquelme $^{\mathrm{b}}$, Joel Sanchez ${ }^{\mathrm{e}}$,Stefan Sandrock ${ }^{\mathrm{a}}$, Ralf Siebenmorgen ${ }^{\mathrm{a}}$, Jörg Stegmeier ${ }^{\mathrm{a}}$, Alain Smette ${ }^{\mathrm{b}}$, \\ Julian Taylor ${ }^{\mathrm{a}}$, Mario van den Ancker ${ }^{\mathrm{a}}$, Guillermo Valdes ${ }^{\mathrm{b}}$, Lars Venema ${ }^{\mathrm{f}}$ \\ ${ }^{a}$ European Southern Observatory, Karl-Schwarzschild-Str. 2, 85748 Garching, Germany; \\ ${ }^{\mathrm{b}}$ European Southern Observatory, Alonso de Córdova 3107, Vitacura, Santiago de Chile, Chile; \\ ${ }^{\mathrm{c} J e t}$ Propulsion Laboratory, California Institute of Technology, E. California Blvd, Pasadena, USA \\ ${ }^{\mathrm{d}}$ CEA Saclay, DSM/DAPNIA/Service d'Astrophysique, 91191 Gif-sur-Yvette, France \\ ${ }^{\mathrm{e}}$ Max Planck Institute for Astronomy, Königstuhl 17, 69117 Heidelberg, Germany \\ ${ }^{\mathrm{f}}$ ASTRON, Oude Hoogeveensedijk 4, 7991 PD Dwingeloo, The Netherlands
}

\begin{abstract}
We present an overview of the VISIR instrument after its upgrade and return to science operations. VISIR is the midinfrared imager and spectrograph at ESO's VLT. The project team is comprised of ESO staff and members of the original VISIR consortium: CEA Saclay and ASTRON. The project plan was based on input from the ESO user community with the goal of enhancing the scientific performance and efficiency of VISIR by a combination of measures: installation of improved hardware, optimization of instrument operations and software support. The cornerstone of the upgrade is the $1 \mathrm{k}$ by $1 \mathrm{k}$ Si:As AQUARIUS detector array manufactured by Raytheon. In addition, a new prism spectroscopic mode covers the whole N-band in a single observation. Finally, new scientific capabilities for high resolution and high-contrast imaging are offered by sub-aperture mask and coronagraphic modes. In order to make optimal use of favourable atmospheric conditions, a water vapour monitor has been deployed on Paranal, allowing for real-time decisions and the introduction of a user-defined constraint on water vapour. During the commissioning in 2012, it was found that the on-sky sensitivity of the AQUARIUS detector was significantly below expectations. Extensive testing of the detector arrays in the laboratory and on-sky enabled us to diagnose the cause for the shortcoming of the detector as excess low frequency noise. It is inherent to the design chosen for this detector and cannot be remedied by changing the detector set-up. Since this is a form of correlated noise, its impact can be limited by modulating the scene recorded by the detector. After careful analysis, we have implemented fast (up to $4 \mathrm{~Hz}$ ) chopping with field stabilization using the secondary mirror of the VLT. During commissioning, the upgraded VISIR has been confirmed to be more sensitive than the old instrument, and in particular for low-resolution spectroscopy in the N-band, a gain of a factor 6 is realized in observing efficiency. After overcoming several additional technical problems, VISIR is back in Science Operations since April 2015. In addition an upgrade of the IT infrastructure related to VISIR has been conducted in order to support burst-mode operations. Science Verification of the new modes was performed in Feb 2016. The upgraded VISIR is a powerful instrument providing close to background limited performance for diffraction-limited observations at an 8-m telescope. It offers synergies with facilities such as ALMA, JWST, VLTI and SOFIA, while a wealth of targets is available from survey works like WISE. In addition, it will bring confirmation of the technical readiness and scientific value of several aspects for future mid-IR instrumentation at Extremely Large Telescopes. We also present several lessons learned during the project.
\end{abstract}

Keywords: VISIR, upgrade, ESO VLT, mid-IR, AQUARIUS, excess low frequency noise, chopping, water vapour, lessons learned

*fkerber@eso.org; phone +49 89 32006757; fax +49 89 32006838; www.eso.org

Ground-based and Airborne Instrumentation for Astronomy VI, edited by Christopher J. Evans, Luc Simard, Hideki Takami Proc. of SPIE Vol. 9908, 99080D · @ 2016 SPIE · CCC code: 0277-786X/16/\$18 · doi: 10.1117/12.2232441 


\section{INTRODUCTION}

VISIR is the mid-IR imager and spectrograph at ESO's Very Large Telescope (VLT) ${ }^{1}$. It was built by a French-Dutch consortium (Service d'Astrophysique CEA and ASTRON - PI: P.O. Lagage, Co-PI: J.W. Pel) and has been operational since the end of 2004. It is located at the Cassegrain focus of unit telescope (UT) 3 (Melipal) at VLT on Cerro Paranal $(2635 \mathrm{~m})$ in Northern Chile. The instrument provides diffraction-limited imaging at high sensitivity in the two midinfrared (mid-IR) atmospheric windows: the N-band between 8 to $13 \mu \mathrm{m}$ and the Q-band between 16.5 and $24.5 \mu \mathrm{m}$ (imaging in the M-band, at $4.6 \mu \mathrm{m}$ is now also possible). In addition, it features a long-slit spectrometer with a range of spectral resolutions between 150 and 30,000.

The upgrade of VISIR has been described at previous SPIE conferences ${ }^{2,3,4}$. For convenience some of the relevant information is repeated and then an update on the recent developments will be given.

For technical details of the instrument and its use we refer to the VISIR homepage at ESO: http://www.eso.org/sci/facilities/paranal/instruments/visir/ and to the description of the instrument by the consortium ${ }^{1}$. Updates on the upgrade status are also available on a dedicated web page (http://www.eso.org/sci/facilities/paranal/instruments/visir/upgradeproject.html). ESO issues a call for proposals twice a year; preparation of proposals asking for observing time with VISIR is supported by an exposure time calculator: http://www.eso.org/observing/etc/.

The goals of the upgrade are summarized in the following list:

- Improved performance of existing modes: achieve background limited performance for imaging and lowresolution spectroscopy

- Optimise use of observing time: more efficient N-band spectroscopy, knowledge of atmospheric conditions, in particular precipitable water vapour (PWV)

- Provide new science capabilities: new modes for high contrast and high resolution imaging

- Expand support of data analysis software in line with enhanced capabilities and ESO policies

- Conduct upgrade in close collaboration with Paranal and partners from the original consortium

\section{AQUARIUS DETECTOR ARRAY}

ESO funded the development of a new Si:As Impurity Band Conduction (IBC) array, named AQUARIUS, at Raytheon Vision Systems, Santa Barbara, USA. Five science devices, an engineering grade and multiplexers have been delivered to ESO as part of this contract. AQUARIUS has $1024 \times 1024$ pixels, each $30 \mu \mathrm{m}^{2}$ in size and is therefore approximately five to six times larger in area than the previous generation of high background mid-IR detectors.

The architecture of the detector is such that it is split into two areas each of 512 rows and 1024 columns, at the top and bottom of the device. Each area has 32 outputs, such that, each output is configured to read out $32 \times 512$ pixels, all 64 outputs from the two areas being read in parallel. This readout scheme also allows for 16 outputs rather than 64 to simplify system design for low background applications. With this multiplexer configuration it is possible to read the detector out at $150 \mathrm{~Hz}$ frame rates, each output operational at $3 \mathrm{MHz}$ pixel rates. A windowed readout is possible, where a user selectable number of rows can be read, from the center outwards, with the remaining rows reset automatically. For example, a 1024 x 150 sized window, centred in the middle of the device, can be readout at $1 \mathrm{kHz}$ frame rates. There is no advantage to windowing in the column direction since all outputs run in parallel. Windowed read-out is routinely used in operations. A full description of the AQUARIUS array and its performance is given by Ives et al. ${ }^{5}$. 


\section{COMMISSIONING AND PERFORMANCE PROBLEMS}

After laboratory testing showing very satisfactory results, the AQUARIUS detector was known to have much better stability and cosmetics than the old detectors. This was confirmed on-sky but unfortunately, during the first commissioning (mid-2012), the sensitivity was found to be a factor 2-3 worse than the one with the old detector. In this state VISIR evidently was not meeting the goals of the upgrade. After evaluating the situation and the options, we decided that VISIR was not fit for science use, and ESO informed the community accordingly. Since the manufacturer only provided very limited support, we than initiated a vigorous test program to identify the cause of the low performance. This entailed an upgrade to our lab test facility in order to simulate conditions on-sky as closely as possible and to validate proposed mitigation options.

For the AQUARIUS detector, there were no reliable quantum efficiency values available. When the commissioning of VISIR showed a lack of sensitivity, we therefore decided, as a first step, to invest dedicated effort in evaluating the detector quantum efficiency. The quantum efficiency of the AQUARIUS detector was found to be $40 \pm 1.5 \%$, a value which is within expectations ${ }^{4,5}$. Thus, an intrinsically low QE was not causing the observed under-performance.

\subsection{Excess low frequency noise (ELFN)}

As a result of extensive testing, we were able to identify the root cause for the limited sensitivity: excess low frequency noise. This phenomenon was described already 30 years ago by Stapelbroek et al. ${ }^{6}$. The ELFN is a form of correlated noise caused by fluctuations in the space charge induced by ionization/recombination in the blocking layer. It manifests as a memory of photons in subsequent frames. This correlation can be broken by modulating the scene (sources and background) seen by the detector at sufficiently high speed. Since the AQUARIUS array was originally developed for space applications with extremely low background (JWST/MIRI), it has a thick blocking layer, and hence this design results in significant ELFN in high background conditions, which has a massive impact on sensitivity in the groundbased (high background) case as for VISIR (Fig. 1). This was not properly accounted for in the design of the Si:As detector material hybridized on the AQUARIUS multiplexer in the framework of the ESO contract. A full analysis is given in Ives et al. ${ }^{5}$. It is important to note that for future ground-based applications, a better design will be warranted.

\section{MITIGATING THE PROBLEM AND RECOVERING ON-SKY PERFORMANCE}

For VISIR, we were faced with the fact that the detector was not meeting the specifications, and since the ELFN can not be mitigated at the detector level, we had to look for alternative solutions to limit the impact of the ELFN and recover the on-sky performance to the best extent possible. We used a combination of laboratory tests and on-sky measurements (Aug 2013) in order to evaluate the options and their merit and feasibility.

\subsection{External chopper}

One obvious solution would have been an external chopper. We studied two concepts for an external chopper: post-focal chopping in the pupil plane, and using a Dicke switch. The first one would be able to provide chopping frequencies up to $20 \mathrm{~Hz}$ but turned out to be impossible to implement because of mass and volume constraints at the VLT Cassegrain focus. The Dicke switch would be limited to less than $5 \mathrm{~Hz}$, and it was found that chopping at such a frequency would be easier to implement in a different way (section 4.3). It is worth noting that in the METIS instrument design for the EELT, a cryogenic chopper is foreseen. Details of the development and characterization of such a cryogenic device was presented at a previous SPIE conference ${ }^{7}$.

\subsection{Drift-scanning}

For drift-scanning the object is moved across the field of view during the observation, hence the scene observed by the detector gets modulated by this motion. The effectiveness of this approach for the AQUARIUS array has been demonstrated during on-sky testing in Aug 2013 (see Fig. 1, lower right). We decided to not implement this method at the VLT because in order to use it in a controlled and flexible way it would have required a major modification of the 
telescope control system. The method is interesting though and is being pursued in the context of future mid-IR instrumentation for the E-ELT by Heikamp et al. .

\subsection{Faster chopping with the VLT's secondary mirror}

From the laboratory tests and on-sky testing, we found that faster chopping will limit and control the impact of the ELFN. A frequency of 2-3 Hz would be adequate to achieve better performance than with the old DRS detector (Fig. 1), and background-limited performance should be within reach or many filters in good seeing conditions.

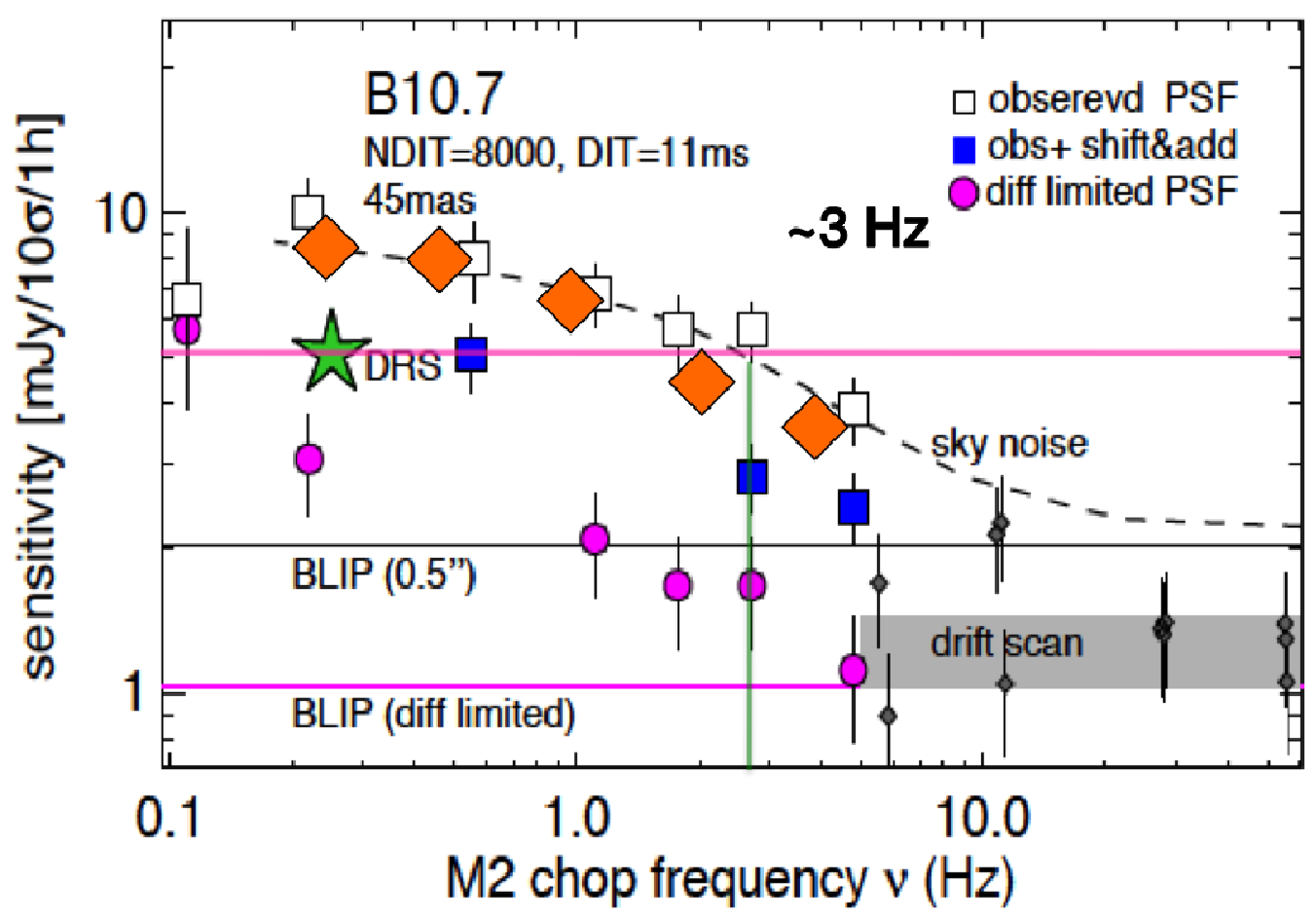

Figure 1. VISIR sensitivities as a function of chopping frequency. Burst mode data were obtained in the B10.7 filter on the standard star HD198048. Predicted sensitivities were deduced from three types of data processing: i) standard imaging mode observation; these are affected by seeing and image quality problems (open squares); ii) applying a shift-and-add correction of the jittered image (blue squares); and iii) estimating the noise from the background. The sensitivity estimate approximates the best possible diffraction-limited performance (magenta circles). For comparison, the median sensitivity of B10.7 filter with the DRS detector is marked by a green star. Sensitivities derived from drift scans located at equivalent chopping frequencies (derived from the scan speed) are shown as grey dots. The grey shaded area gives their 1-sigma range. Error bars reflect $1 \sigma$ uncertainties and are derived from the variance of the noise. The sky noise extracted from other measurements is scaled to match the $1 \mathrm{~Hz}$ data point. The dashed line gives the expected sensitivity gain with higher chopping frequency. Theoretical sensitivity limits (BLIP) are shown for 0.5" seeing (solid line) and for diffraction limited observations (magenta line). The actual measured performance (mediocre seeing) with the faster chopping implemented is given by orange diamonds. The measured performance is in good agreement with the prediction (dashed line) and confirms the increase in sensitivity with higher chopping frequency.

Hence, faster chopping was adopted as primary option for VISIR upgrade to mitigate the effect of ELFN and recover onsky sensitivity. The technical implementation of faster chopping involved a number of changes to the set up of UT3. The technical CDD (TCCD) responsible for the auto-guiding was up for replacement at the UT3 Cassegrain as part of the Paranal programme to address obsolescence. This exchange was advanced for the VISIR upgrade to take advantage of the frame transfer capability of the TCCD (CCD57-10 by e2VTM). Hence the read-out becomes a continuous process resulting in a significantly increased efficiency of above $87 \%$, even at fast rates. The other aspect was to optimise the 
field-stabilisation (FS) loop for faster chopping. A simulation model of the FS loop was built and the dynamic response of M2 was measured on UT1 by injecting test signals and logging data. The closed loop controller was found to become unstable at high TCCD frame rates. An alternative tuning was calculated in simulation and validated experimentally, allowing chopping up to $4 \mathrm{~Hz}$ on sky. Details of the work have been described at a previous conference ${ }^{4}$.

Figure 1 also illustrates the predicted and observed performance of the scanning and faster chopping with the VLT's M2. The predicted performance is based on observations simulating the two mitigation options. The measured performance is in good agreement with the prediction (dashed line) and confirms the increase in sensitivity with higher chopping frequency. On-sky sensitivity is better than with the old detector for higher than $3 \mathrm{~Hz} \mathrm{M} 2$ chopping. Better performance can be achieved for burst mode observations and shift and add.

\section{VISIR UPGRADE - NGC DETECTOR CONTROL SYSTEM}

NGC is the ESO New General detector Controller ${ }^{9,10}$, designed to handle the detectors of both optical and infrared instruments, for scientific imaging as well as advanced signal sensing applications. In the scope of the VISIR upgrade project the NGC replaces its predecessor IRACE $E^{11}$.

In VISIR, the controller drives the two AQUARIUS high flux $1 \mathrm{k} \times 1 \mathrm{k} \mathrm{Si}$ :As arrays, which are deployed for the midinfrared imaging and spectroscopy applications. Each array has 64 parallel video output channels and can be read out at frame rates of up to $150 \mathrm{~Hz}$ (Fig. 2). The read-out is synchronized with a chopper running at frequencies of up to $5 \mathrm{~Hz}$. Figure 3 shows a timing example for Simple-Read-Reset mode (SRR). The arrays are not operated simultaneously, but the system is configured to allow rapid switching between imaging and spectroscopy observations.

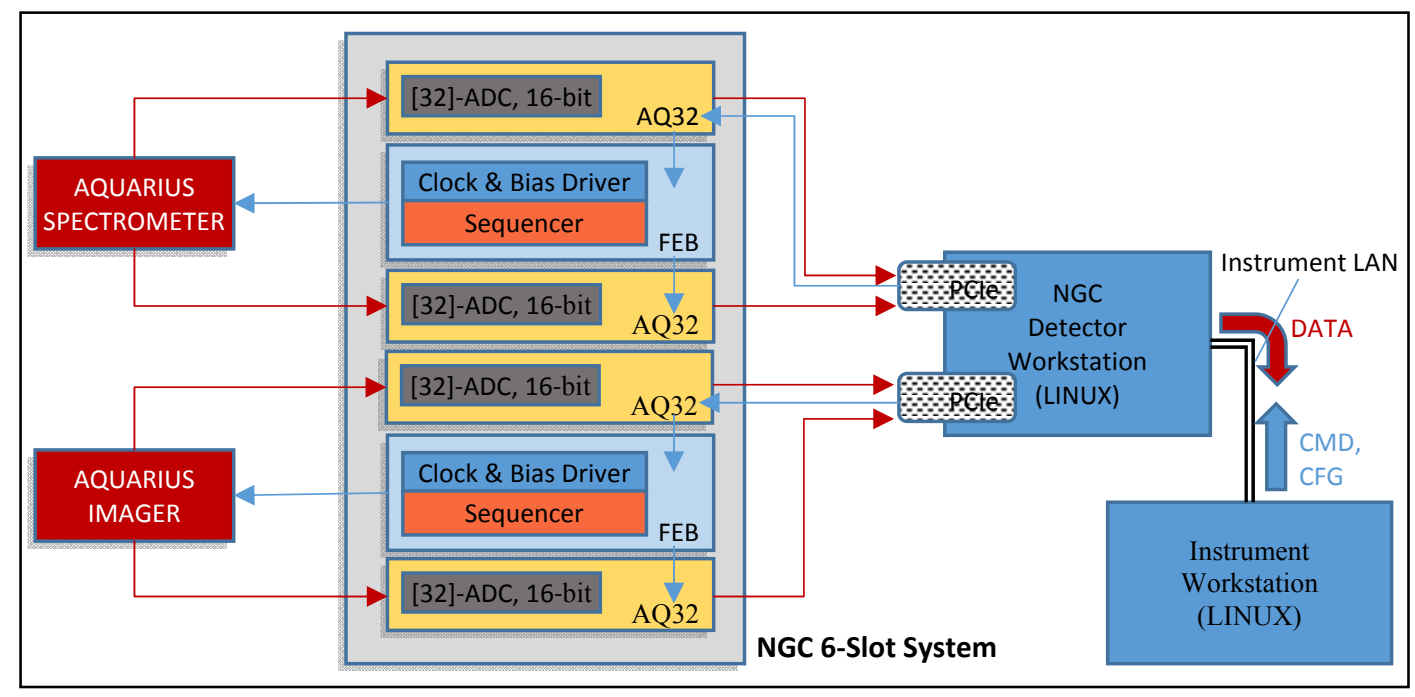

Figure 2. System Architecture of the NGC controller

The data acquisition, which is running on the NGC detector workstation, is a multi-threaded pre-processing framework with a high task concurrency. The framework produces a configurable number of frame types: "raw frame" (DIT), "chopper half-cycle frames" (HCYCLE1,2), "chopped integration" (INT). Each frame type can be sent to a real-time display for quick look. An exposure is finished when the "INT" frame, assembling several chop cycles, has been received on the instrument workstation. Optionally, for further processing in the data pipeline, the averaged frames after each chopping half-cycle can be stored in the multi-extension FITS-file. For lower resolution post-processing, it is sufficient to save the overall average for each chopper position. 


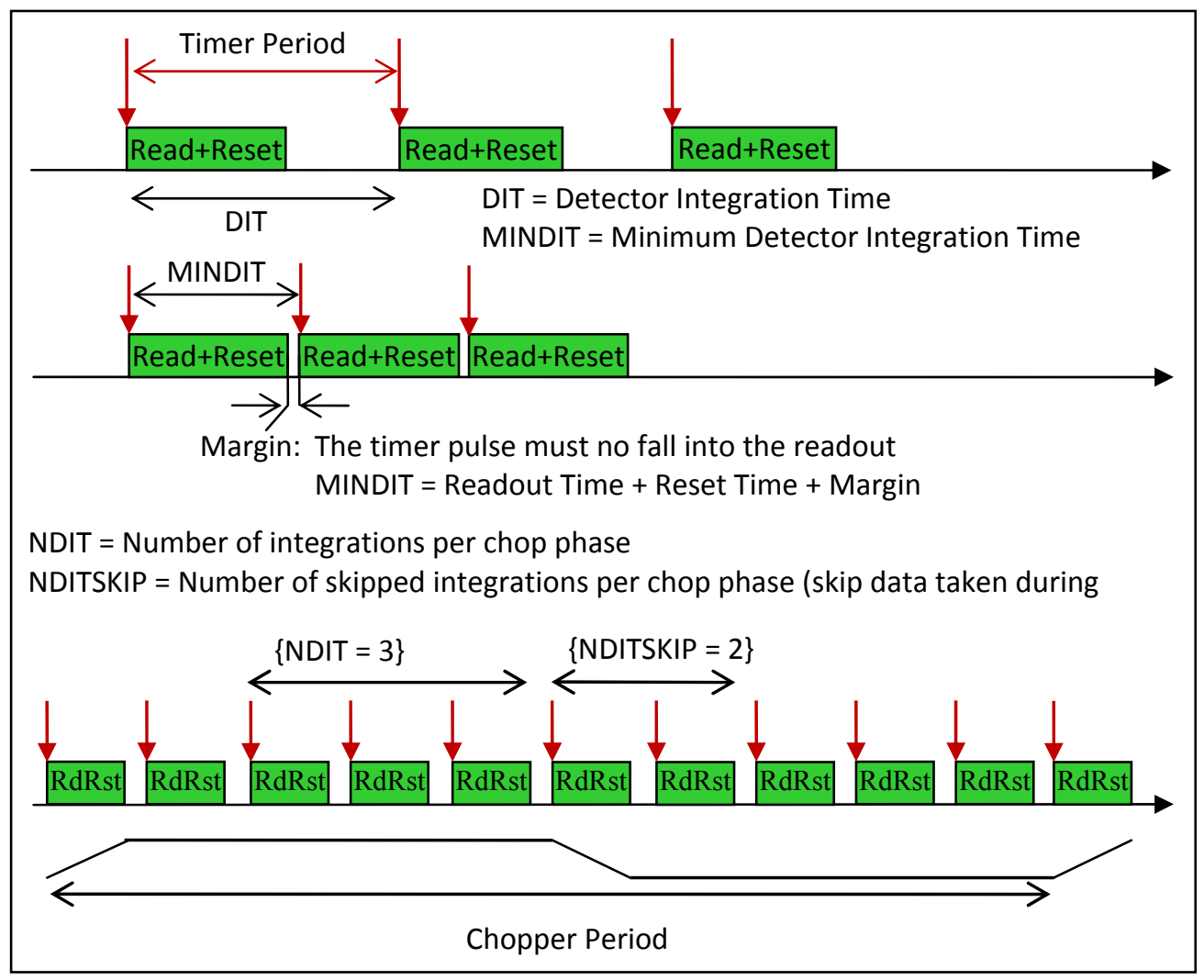

Figure 3. Diagram illustrating the chopper synchronization per read-out

To enable full post-processing capabilities in certain modes, the system needs to store the raw data of a full exposure covering multiple chop cycles. The NGC detector control software provides an optimized "burst-mode", which allows to buffer large amounts of raw data at maximum data rate. The sustained scatter/gather-DMA transfer remains synchronized with the chopper, while pipelining the data through the pre-processor to the output queue of a singlewriter/multiple-reader publishing gateway. To minimize the exposure latency, the output queue is already flushed while recording the burst data. The mechanism guarantees a lossless acquisition sequence up to the limit of the RAM installed on the detector workstation. Once the burst is completed, the standard acquisition is resumed without losing the synchronization. The burst-mode also helps to analyze the behavior of many involved sub-systems, such as the telescope's field stabilization facilities. The AQUARIUS detector topology allows window readouts only in Y-direction. To further reduce the data volume, which may become significantly high in "burst-mode", the NGC pre-processing framework provides soft-windowing methods both in X-and Y-direction for all modes and frame types.

\section{SCIENCE PERFORMANCE}

VISIR was re-commissioned during the period Oct 2014 to April 2015 and was declared ready for science operations immediately after that. The overall performance of the old operation modes (imaging and spectroscopy) improved. Using faster M2 chopping, the imaging sensitivities are considerably better than with the old VISIR (Figure 1, 4 \& 6), despite the shortcoming of the AQUARIUS detector. Although not initially planned, it was possible to insert and successfully test an M-band filter $(\sim 4.6 \mu \mathrm{m})$ that now can be used for imaging as well. As before, the burst mode provides excellent image quality but now benefits a lot from the superior detector cosmetics of the AQUARIUS (Figure 5). Science Verification of the new modes coronagraphy ${ }^{12,13}$ (Fig. 7) and SAM ${ }^{14,15}$ (Fig. 8) was performed in Feb and Mar $2016^{16}$. 


\subsection{Imaging sensitivity}

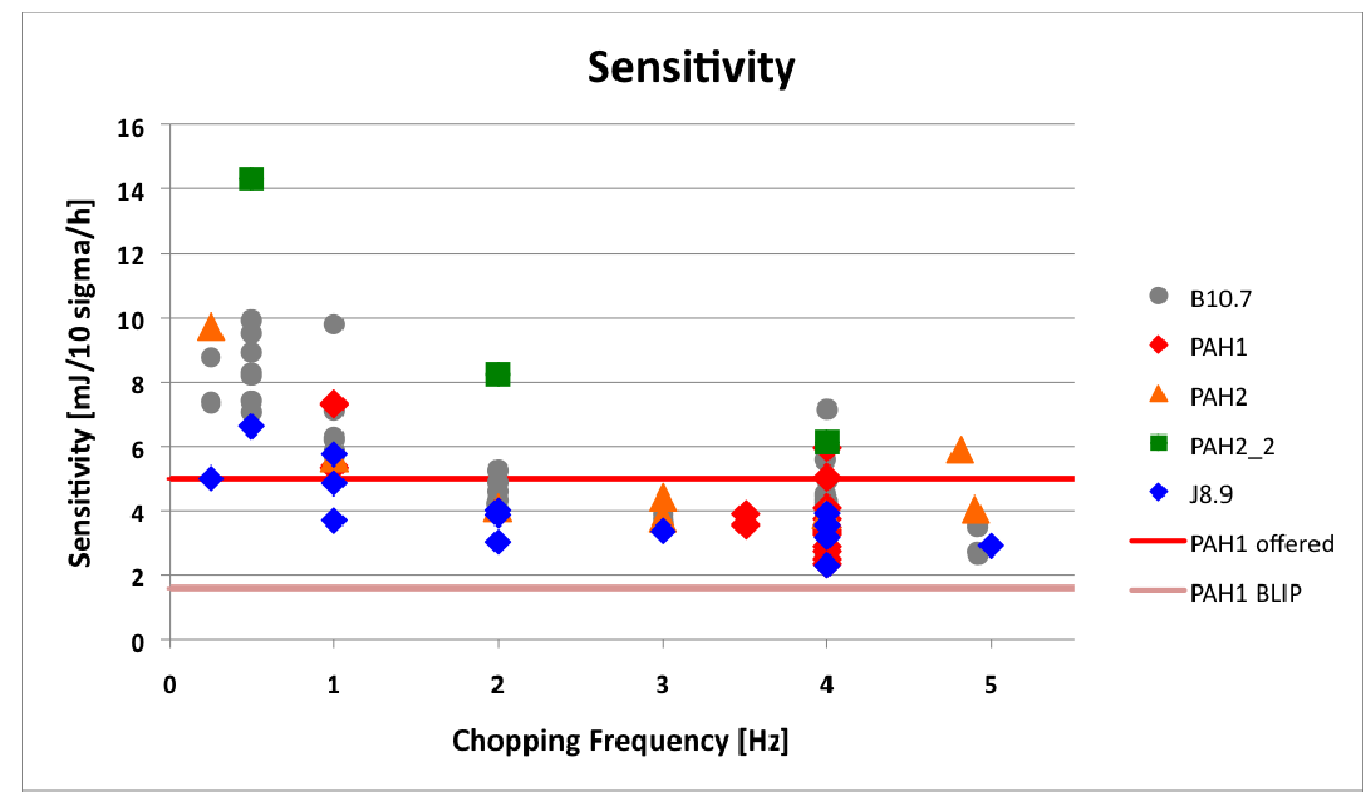

Figure 4. Sensitivities in imaging for different filters and chopping frequencies measured on sky with calibrator stars during the commissioning. The top plot shows the individual measurements for different N-band filters and the theoretical limit as straight light-colored line. A very similar behaviour and performance with respect to the old VISIR is found for the Q-band filters.

\subsection{Image quality burst mode}

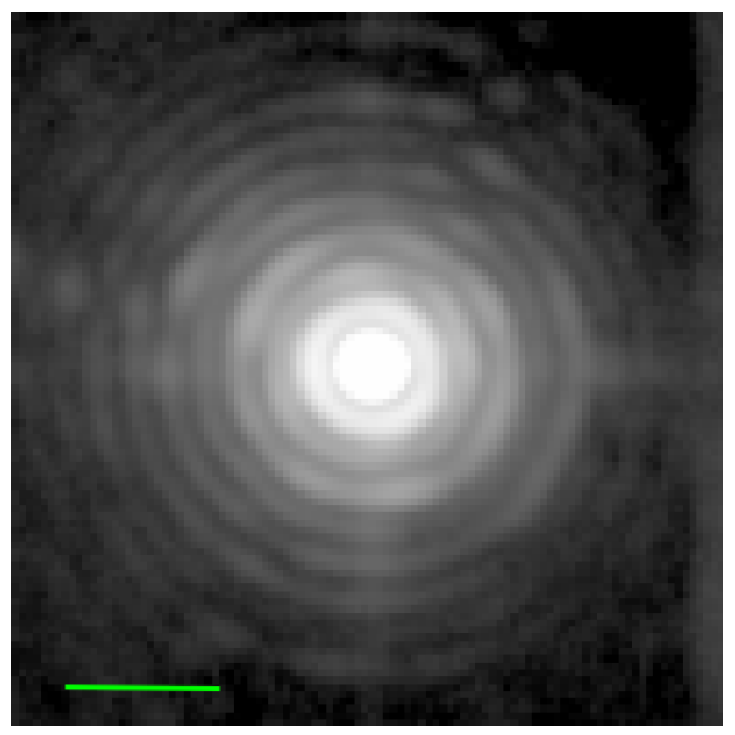

Figure 5. Point spread function for a bright star for a short sequence of burst mode imaging. Shift and add of the individual frames leads to a superb image quality at the diffraction limit, as evident by the many Airy rings. The scaling is logarithmic and the green bar indicates 1 arcsec on sky. The target was of the bright star HD 45348 ( 150Jy), observed with a DIT of $0.01 \mathrm{~s}$ and NDIT $=5000$ frames in the PAH1 filter. 


\subsection{Spectroscopic sensitivity}

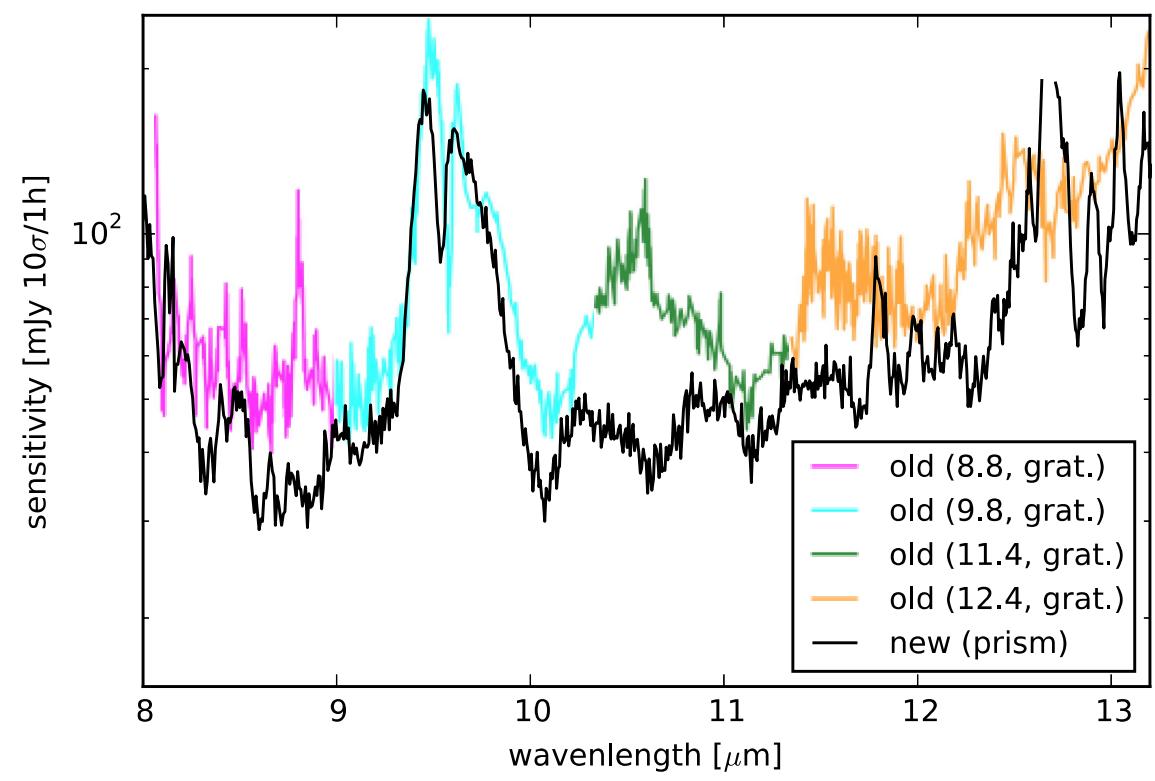

Figure 6. Spectroscopic sensitivity in the low-resolution mode for the N-band. The colored lines show the median sensitivity of the pre-upgrade VISIR, while the colors denote the different grating settings that were necessary to cover the full accessible range. The black line shows the median sensitivity of the upgraded VISIR with the prism (one shot) as obtained during the commissioning.

\subsection{Coronagraphy with the Annular Groove Phase Mask (AGPM)}
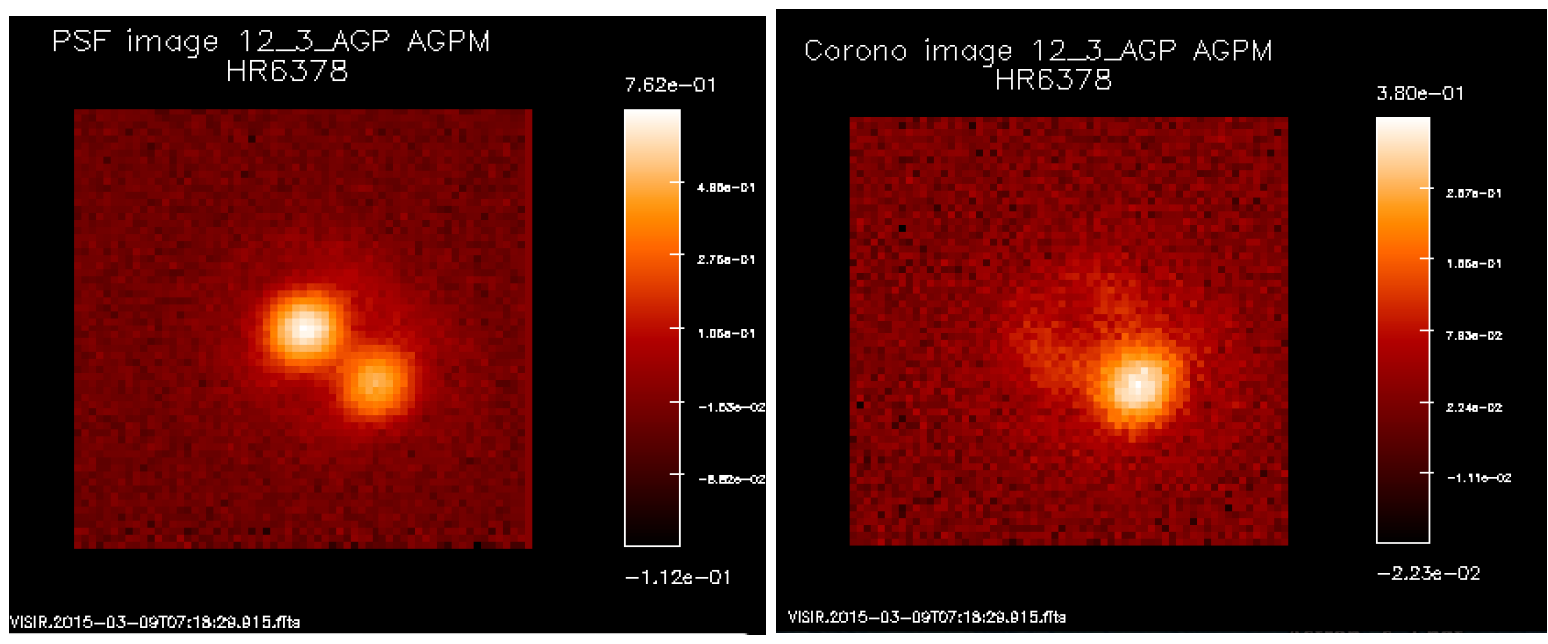

Figure 7. Comparison of double star HR 6378 imaged directly (left) and with annular grove phase mask coronagraph (right) at $12.3 \mu \mathrm{m}$. The coronagraph was centered on the brighter primary in this case. 
The annular groove phase mask (AGPM) coronagraph ${ }^{12,13}$ point source extinction (or attenuation) has been measured on several standard stars. This quantity depends strongly on the seeing conditions. The seeing value was about 0.9 arcsec (visible) during the observations, so at $10 \mu \mathrm{m}$ on a $8 \mathrm{~m}$-class telescope, we consider to have a significant error due to the seeing (diffraction starts to dominate at a seeing below 0.7 arcsec). We measure a peak attenuation of $40 \pm 2$ and integrated extinction of a factor of $3.8 \pm 0.1$. Further performance tests in good seeing conditions are necessary to fully assess the performance of the coronagraph.

\subsection{Sparse Aperture Masking}
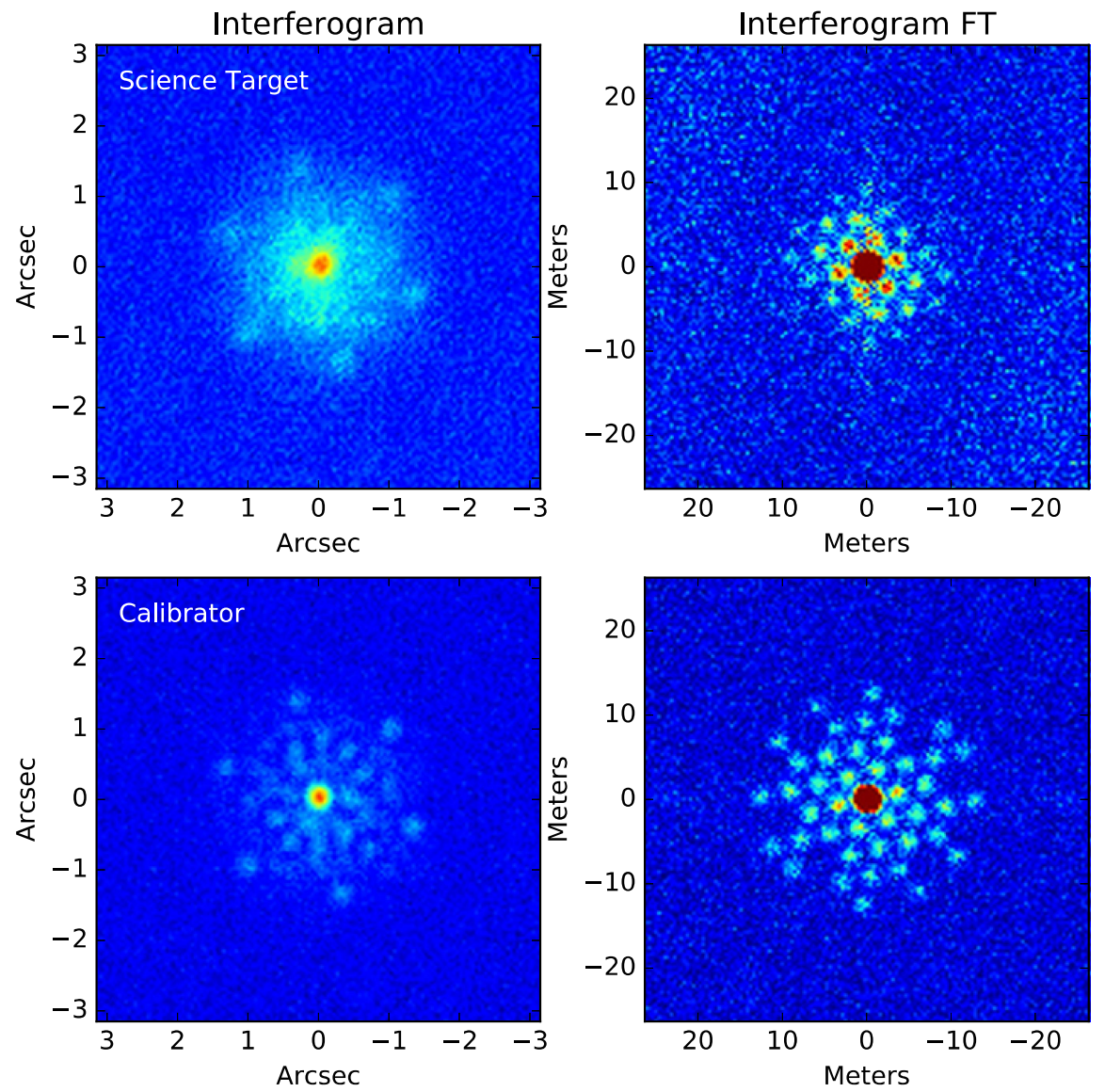

Figure 8. Interferogram at $10.5 \mu \mathrm{m}$ of the massive young stellar object NGC 3603 IRS9A (top) and the calibrator star HD 96918 (bottom) obtained using VISIR's new SAM mode ${ }^{14,15}$ during science verification. The fuzzier appearance of NGC 3603 IRS9A with respect to the calibrator star demonstrates that the science target is resolved at the baselines corresponding to the sub-apertures of the SAM mask [figure courtesy of Joel Sanchez (MPIA), also see Asmus et al. ${ }^{16}$ ]. 


\section{SUPPORT OF SCIENCE OPERATIONS: ATMOSPHERIC PROPERTIES}

\subsection{Precipitable water vapour as a user-defined constraint}

A water vapour monitor has been permanently deployed at ESO's Paranal observatory as part of the VISIR upgrade project. The Low Humidity And Temperature PROfiling microwave radiometer (LHATPRO), manufactured by Radiometer Physics GmbH (RPG). measures several channels across the strong water vapour emission line at $183 \mathrm{GHz}$, necessary for resolving the low levels of precipitable water vapour (PWV) that are prevalent on Paranal ${ }^{17}$ (median $\sim 2.5$ $\mathrm{mm}$ ). Details of the instrument and its operations have been presented at a previous SPIE ${ }^{18}$. The introduction of PWV as a user defined constraint - similar to seeing or sky transparency in the optical - provides the opportunity to optimize the scientific return of infrared instruments like VISIR by matching the PWV needs of each observation carried out in service mode to the actual conditions measured in real-time by the PWV monitor. Furthermore, the addition of a realtime PWV monitoring capability to Paranal infrastructure will enable qualitatively new types of science projects that require conditions of particularly low $\mathrm{PWV}^{19}$ to be successfully executed.

\subsection{Mid-IR Image Quality - predicted from optical seeing versus observed}

Van den Anker et al. ${ }^{20}$ present an analysis of the image quality observed with VISIR and show that there is a systematic difference of about 0.1 " between the $\mathrm{N}$-band image quality predictions of the VISIR ETC based on the optical seeing and the realized image quality. The fact that (1) the measured image quality is not constant, (2) the image quality achieved by VISIR in the N-band is close to that achieved by other mid-IR instruments on 8-m class telescopes (3) the in the Q-band measured image quality is indeed close to the diffraction limit, and (4) the image quality in the N-band shows the expected $\mathrm{x}^{0.6}$ dependence on airmass, shows that this difference is due to the effects of the earth's atmosphere and not due to image degradation within the instrument. This difference in predicted and achieved image quality has important implications for the scheduling of VISIR service-mode observations and van den Anker et al. ${ }^{20}$ make a recommendation to adopt an improved description of the mid-IR seeing for science operations with VISIR.

\section{LESSONS LEARNED}

The upgrade of VISIR proved to be significantly more complex than anticipated and in particular took longer to implement. It would be inappropriate to consider this outcome to be bad luck. Thus, we summarise a few lessons for future consideration in the following.

The main reason was the deficient on-sky performance of the AQUARIUS detector array as a result of a design that is unsuitable for high background, hence ground-based astronomical applications. It required a major effort to diagnose the root cause of ELFN and additional effort to find and implement a suitable mitigation since it couldn't be remedied at the detector level.

It is safe to say that upgrading, as well as developing, astronomical instruments is an intrinsically risky undertaking because essentially all of them are prototypes. This needs to be managed by active risk management for known, and contingency, for unknown risks.

A careful assessment of the technology readiness levels of (sub-)systems should be a must at the approval stage for any project. In our case, the planning was too optimistic in this respect based on the fact that the AQUARIUS had been developed and tested for space application indicating good readiness, which however did not apply to the ground-based case.

Best effort contracts are inherently very risky. The detector did not meet the specification due to a flawed design; it is intriguing to see that an effect described more than 30 years ago remained obscure even to the detector experts. Support by the manufacturer was very limited, an aspect that can be expected to differ for a standard contract.

In the case of technical problems, in-house capabilities are essential. In our case, the expertise of our detector group was crucial in diagnosing the problem with the help of experts from the cryo-vacuum group using existing and upgraded laboratory facilities. Experts for detector and telescope control found ingenious solutions to mitigate the ELFN problem. 
On the practical level, proper as-built drawings and documents are most helpful in avoiding pitfalls during the upgrade and instrument interventions.

The complexity of control software for telescope, detector and instruments alike require a careful definition of the necessary changes for the upgrade and a close collaboration between the astronomers and software engineers. The effort here is easily underestimated and requires proper buffer and contingency.

Finally, we benefitted greatly from the excellent collaboration between the project team and Paranal observatory staff.

\section{OUTLOOK}

One of the drivers for the VISIR upgrade was that the user demand for VISIR prior to its upgrade was clearly lower than other instruments installed at the VLT. Fig. 9 shows the evolution of the number of submitted observing proposals per semester. Whereas prior to the VISIR upgrade (ESO periods 86-88), the number of proposals per semester was always less than 30 (for comparison: the most in-demand instruments at the VLT, FORS2, MUSE and X-SHOOTER average more than 100 proposals per semester), in the periods leading up to the upgrade the demand rose sharply to a maximum of 64 proposals in ESO period 91, comparable to other successful VLT instruments such as NACO, SPHERE and SINFONI.

After VISIR was taken out of operations for several periods to resolve the issues with the new Aquarius detector, demand came back at a lower level (around 30 proposals per semester), but has increased since to a steady level of around 45 proposals per semester (Fig. 9). The requested number of more than 800 hours per semester greatly exceeds the available time per instrument, leading to a over-subscription by more than a factor of two (i.e. less than half of the received proposals will be allocated observing time). The above numbers show that when a state-of-the-art mid-IR instrument is offered on a large ground-based telescope, the demand from the user community is similar to that for recent near-IR and optical instruments.

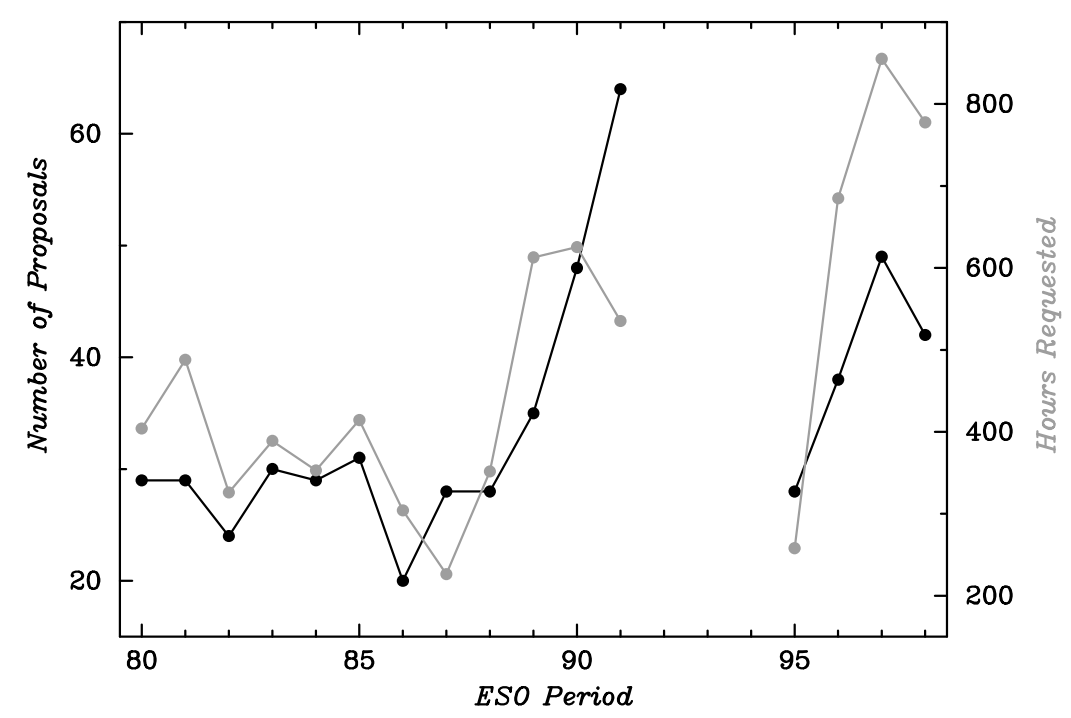

Figure 9. Demand for VISIR observations by the ESO community showing the increase with respect to the old instrument.

Currently, a few open issues remain with VISIR. These pertain to the image quality in standard imaging and fringing in spectroscopic mode. Both are actively being worked on and will be addressed as part of the continuous improvement efforts. In addition, performance for the coronagraphic imaging and SAM can be expected to be improved by a dedicated centering algorithm which is under investigation. For the new science modes performance analysis is still in progress; updated information will be provided to the ESO community. We expect that astronomers will be able to make good use 
of the upgraded VISIR and its capabilities in the future and look forward to exciting scientific results as already highlighted by the science verification observations ${ }^{16}$.

\subsection{Mid-IR sky background}

The availability of LHATPRO now allows for detailed studies of the mid-infrared sky background using LHATPRO and VISIR simultaneously, which will help to find more efficient ways of background removal for future ground-based midinfrared instrumentation like METIS for the E-ELT. We have started such a pilot study observing the sky background variations with high time resolution staring at zenith using the burst mode of VISIR and a custom mode on LHATPRO in the same line-of-sight (Fig. 10).
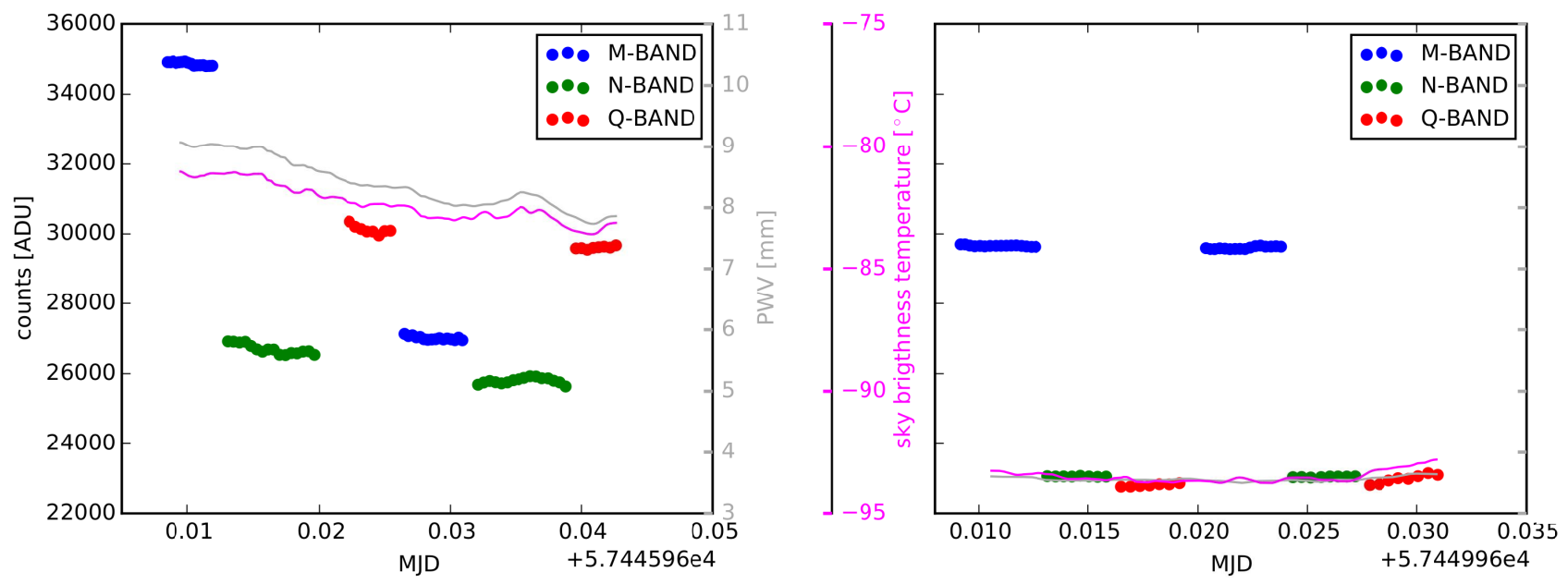

Figure 10. Comparison of the count levels (per DIT) in VISIR and PWV and sky brightness temperature from LHATPRO for two nights during science verification (prog. ID 60.A-9621). The filled circles mark the median count levels of individual burst cubes of 20s length using different filters (M-Band in blue, B10.7 in green and Q2 in red), while the grey and magenta lines show the smoothed PWV and sky brightness temperature curves. The left plot shows the night of Feb. 27, a night with very high PWV and sky temperature, while the right plot shows the night of Mar. 02, a night where PWV and sky brightness temperature being closer to average. It seems that the background levels in VISIR correlate with the PWV and sky temperature. Further detailed analysis will show which bands depend stronger on PWV or the sky brightness temperature.

\section{SUMMARY}

We have presented an overview of the VISIR instrument after its upgrade and return to science operations. We describe several of the problems encountered and the mitigation actions needed to make sure that VISIR upgrade indeed resulted in improved performance. We illustrated the scientific performance of VISIR and highlighted some of the changes in operations providing better support to the user. The upgraded VISIR is a powerful instrument providing close to background limited performance for diffraction-limited observations at an 8-m telescope. This has been recognized by the mid-IR community as indicated by a significantly increased demand for VISIR compared to the period before the upgrade. Hence the title of this contribution.

Acknowledgements: We gratefully acknowledge the support by Paranal staff and the good collaboration. In particular we thank M. Tapia and the staff of the machine shop for their quick and effective support. Great support was already provided by system engineering and science operations. Special thanks go to Prof. Peter Tuthill (The University of Sydney, Australia) for designing the sparse aperture mask. 
Product Disclaimer Certain commercial equipment, instruments, or materials are identified in this report in order to specify the experimental procedure adequately. Such identification is not intended to imply recommendation or endorsement by the European Southern Observatory, nor is it intended to imply that the materials or equipment identified are necessarily the best available for the purpose.

\section{REFERENCES}

[1] Lagage, P. O., Pel, J. W., Authier, M., et al., "Successful commissioning of VISIR: The mid-infrared VLT Instrument," The Messenger 117, 12-16 (2004)

[2] Kerber, F. Käufl, H.U., van den Ancker, M., et al., "Upgrade of VISIR the mid-infrared instrument at the VLT," Proc SPIE 7735, 77357R1-R12 (2010)

[3] Kerber, F. Käufl, H.U., Baksai, P. et al., "VISIR upgrade overview and status," Proc SPIE 8446, 84460E1-E12 (2012)

[4] Kerber, F., Käufl, H.U., Baksai, P. et al., "VISIR upgrade overview and status," Proc SPIE 9147, 91470C1-C12 (2014)

[5] Ives, D., Finger, G., Jakob, G., Beckmann, U. "AQUARIUS, the next generation mid-IR detector for ground based astronomy, an update," Proc SPIE 9154, 91541J-J11 (2014)

[6] Stapelbroek, M. G., Petroff, M. D., Speer, J. J., Bharat, R., "Origin of excess low frequency noise at intermediate infrared backgrounds in BIB detectors," Proc. IRIS Detector, No.2 (1984)

[7] Paalvast, S.L., Jassen, H., Teuwen, M., et al., "Development and characterization of a 2D precision cryogenic chopper for METIS," Proc SPIE 9151, 91510D1-D8 (2014)

[8] Heikamp, S., Brandl, B., Keller, C. U., et al., "Drift scanning technique for mid infrared background subtraction," Proc SPIE 9147, 91479T1-T6 (2014)

[9] Baade, D., et al., "NGC - ESO's New General Detector Controller," The Messenger 136, $20-24$ (2009)

[10] Meyer M., et al., "Detector Acquisition Hardware Designs and Features of NGC (New General detector Controller," http://www.eso.org/sci/meetings/dfa2009/program.html (2009)

[11] Meyer, M., "The ESO Infrared Detector High-Speed Array Control and Processing Electronics IRACE," The Messenger 86, 14-17 (1996)

[12] Rouan, D., Boccaletti, A., Baudoz, P., Cavarroc, C., Baudrand, J., Reess, J.M., “The Coronagraphic Mode of MIRI/JWST", proceedings of the conference In the Spirit of Bernard Lyot: The Direct Detection of Planets and Circumstellar Disks in the 21st Century, University of California, Berkeley, CA, USA, Ed. Paul Kalas (2007)

[13] Mawet, D., Riaud, P., et al., “Annular Groove Phase Mask Coronagraph” ApJ 633, 1191-1200 (2005)

[14] Tuthill, P. G., Monnier, J. D., Danchi, W. C., “Aperture masking interferometry on the Keck I Telescope: new results from the diffraction limit” Proc. SPIE Vol. 4006, 491-498 (2000)

[15] Monnier, J. D., Tuthill, P. G., Ireland, M., et al., "Mid-Infrared Size Survey of oung Stellar Objects: Description of Keck Segment-Tilting Experiment and Basic Results” ApJ 700, 491-505 (2009)

[16] Asmus, D., van den Ancker, M., Ivanov, V, Käufl, H.U., Kerber, F., Leibundgut, B., Mehner, A., Momany, Y., Pantin, E., Tristram K.W. "VISIR Upgrade Science Verification,” The Messenger 164, in press

[17] Querel, R.R., Naylor, D.A., Kerber, F. "Spectroscopic Determination of Atmospheric Water Vapor,“ PASP 123, 222-229 (2011)

[18] Kerber, F., Rose, T., Chacon, A., et al. "A water vapour monitor at Paranal Observatory," in SPIE Conference Series 8446, 84463N1-N12 (2012)

[19] Kerber, F., Querel, R. R., Rondanelli, R. et al. "An episode of extremely low precipitable water vapour over Paranal observatory," MNRAS 439, 247-255 (2014)

[20] Van den Ancker, M.E., Asmus, D., Hummel, C., et al., "Investigating the effect of atmospheric turbulence on mid-IR data quality with VISIR," in SPIE Conference Series 9910, in press (2016) 